\title{
Beyond a Quartic Polynomial Modeling of the DNA Double-Helix Genetic Code
}

\author{
Hans Hermann Otto \\ Materials Science and Crystallography, Clausthal University of Technology, Clausthal-Zellerfeld, Germany \\ Email: hhermann.otto@web.de
}

How to cite this paper: Otto, H.H. (2021) Beyond a Quartic Polynomial Modeling of the DNA Double-Helix Genetic Code. Journal of Applied Mathematics and Physics, 9, 2558-2577.

https://doi.org/10.4236/jamp.2021.910165

Received: September 14, 2021

Accepted: October 26, 2021

Published: October 29, 2021

Copyright $\odot 2021$ by author(s) and Scientific Research Publishing Inc. This work is licensed under the Creative Commons Attribution International License (CC BY 4.0).

http://creativecommons.org/licenses/by/4.0/

\section{(c) (i) Open Access}

\begin{abstract}
By combination of finite number theory and quantum information, the complete quantum information in the $D N A$ genetic code has been made likely by Planat et al. (2020). In the present contribution a varied quartic polynomial contrasting the polynomial used by Planat et al. is proposed that considered apart from the golden mean also the fifth power of this dominant number of nature to adapt the code information. The suggested polynomial is denoted as $g(x)=x^{4}-x^{3}-\left(4-\varphi^{2}\right) x^{2}+\left(4-\varphi^{2}\right) x+1$, where $\varphi=\frac{\sqrt{5}-1}{2}$ is the golden
\end{abstract} mean. Its roots are changed to more golden mean based ones in comparison to the Planat polynomial. The new coefficients $4-\varphi^{2}$ instead of 4 would implement the fifth power of the golden mean indirectly applying

$4-\varphi^{2}=3+\varphi=\sqrt{13+\varphi^{5}}=\sqrt{2+\varphi^{-5}}=3.6180 \cdots$. As an outlook, it should be emphesized that the connection between genetic code and resonance code of the DNA may lead us to a full understanding of how nature stores and processes compacted information and what indeed is consciousness linking everything with each other suggestedly mediated by all-pervasive dark constituents of matter respectively energy. The number-theoretical approach to $D N A$ coding leads to the question about the helical structure of the electron.

\section{Keywords}

DNA Genetic Code, DNA Resonance Code, Qartic Polymial, Golden Mean, Silver Mean, Fifth Power of the Golden Mean, Fiboacci Number 13, $\alpha$-Helix, Icosahedron Equation, Number Theory, Quantum Computation, Consciousness, Dark Energy, Electron's Structure

\section{Introduction}

In a more literary parlance, the genetic code of $D N A$ is the very language of life, 
made up of four nucleotides ( $\mathrm{A}=$ adenine, $\mathrm{C}=$ cytosine, $\mathrm{G}=$ guanine, $\mathrm{T}=$ thymine) combined to 64 three-letter words, the codons. The code can be read by cellular complexes, the ribosomes, and the information translated to produce proteins. In a brilliant contribution Planat et al. set out unrevealing the complete quantum information in the $D N A$ genetic code by combining finite number theory and quantum information [1]. The cited authors have used a quartic polynomial containing the golden mean $\varphi$ in its structure (see Appendix). Intuitively, the present author suspects that in addition to the golden mean, also the fifth power of this number should play an important role in mapping the genetic code owed to the fact that this number is observed extremely frequently in nature, covering examples from microscopic to cosmic scale [2] [3] [4] [5]. So, one may argue that this feature should also be found in some way in the genetic code. A little varied quartic polynomial can deliver such an implementation of $\varphi^{5}$ by replacing the starting polynomial coefficients adequately. This was described in Chapter 2. In times of a viral pandemic, it is essential to know the genetic code of $D N A$ respectively surface proteins in order to effectively block the replication capacity of any pathogenic virus via this route. A further practical aspect of research in this direction is to pave the way for quantum computation under ambient conditions. Recently, a very creative review for understanding of what may be important when dealing with such suggestions has been given by Savelyev et al. connecting the DNA resonance code to the neural code [6]. When translating such connections in the parlance of phase transition, again one comes back to the golden ratio respectively its fifth power [4], both considered as fundamental numbers of nature.

The presented contribution is a short excerpt from a more comprehensive review coming soon about new aspects of helical structures in general [7]. Therein a didactic explanation for the characteristic diffraction pattern of a helix (doublehelix) will be presented in detail.

Finally, the algebraic-geometrical approach to the code of the $D N A$ and secondary protein structures leads to the question what structure a fundamental particle like the electron has.

In the Appendix some supplements were made comparing the just mentioned quartic polynomials with double well potential curves respectively the Hardy probability function [2].

\section{Varying the Quartic Polynomial $f(x)$}

For a better understanding of the solution of polynomial equations, the recent work of Tehrani [8] is highly recommended.

The starting Planat quartic polynomial $(P Q P)$ given in [1] is

$$
f(x)=x^{4}-x^{3}-4 x^{2}+4 x+1
$$

Its curve is illustrated in Figure 1 and its four roots (zeros) are presented in Table 1 according to [1]. What happens, if the polynomial would be slightly varied by replacing the coefficients 4 by $4-\varphi^{2}=3+\varphi$, where $\varphi$ is the golden 


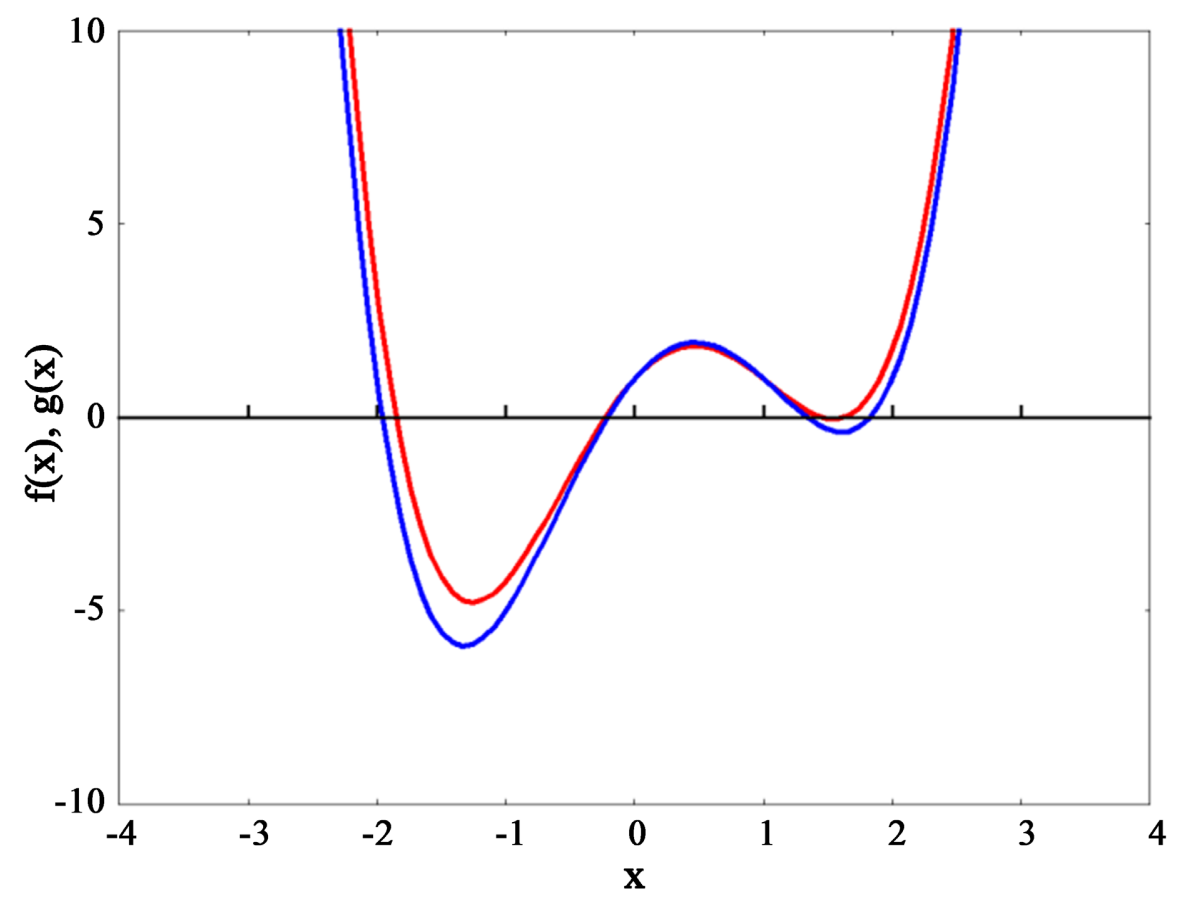

Figure 1. Comparison of the polynomials $f(x)$ (blue) and $g(x)$ (red).

Table 1. Roots of the quartic polynomials $f(x)$ and $g(x)$.

\begin{tabular}{ccccc}
\hline Roots & \multicolumn{2}{c}{$f(x)$} & \multicolumn{2}{c}{$g(x)$} \\
\hline$r_{1}$ & $-2 \cos \left(\frac{\pi}{15}\right)$ & $-1.956295 \ldots$ & $\approx-\left(\varphi^{-1}+\varphi^{3}\right)$ & $-1.8516618 \ldots$ \\
$r_{3}$ & $-2 \cos \left(\frac{7 \pi}{15}\right)$ & $-0.209056 \ldots$ & $\approx-\varphi^{3}=-0.2360679 \ldots$ & $-0.2283086 \ldots$ \\
$r_{2}$ & $-2 \cos \left(\frac{11 \pi}{15}\right)$ & $1.338261 \ldots$ & $-2 \cos \left(\frac{12 \pi}{15}\right)=\varphi^{-1}$ & $1.61803398 \ldots$ \\
$r_{4}$ & $-2 \cos \left(\frac{13 \pi}{15}\right)$ & $1.827091 \ldots$ & & \\
\hline
\end{tabular}

mean: $\varphi=\frac{\sqrt{5}-1}{2} ?$

The coefficients now used possibly represent limits and can be recast by

$$
4-\varphi^{2}=\sqrt{13+\varphi^{5}}=3.6180339887 \cdots
$$

In this way one would implement the fifth power of the golden mean indirectly. The new polynomial is denoted as $g(x)$ and is given by

$$
g(x)=x^{4}-x^{3}-\left(4-\varphi^{2}\right) x^{2}+\left(4-\varphi^{2}\right) x+1
$$

Its roots are changed to more golden mean based ones in comparison to the starting polynomial (see Table 1). Both quartic polynomials can be compared in Figure 1. The curve for $g(x)$ is somewhat flattened and compressed in comparison to the starting curve. 
Remarkably, for $g(x)$ two zeros $r_{2}$ and $r_{4}$ meet at $x=\varphi^{-1}$. This flatten out of the curve around zero may be important, when considering the polynomial as a template for a potential energy model of the $D N A$ double helix and critical behavior. About double well potential important in quantum information theory see Appendix. The maximum of $g(x)$ at $x=0.4661 \approx 2 \varphi^{3}$ has, similar to the $f(x)$ polynomial, a value of

$$
g_{\max }=1.846288 \cdots \approx \varphi^{-1}+\varphi^{3} .
$$

The effect of the varying coefficients is a pronounced presence of the golden mean hierarchy compared to the starting polynomial. Consequences of the variation of the quartic polynomial with respect to group theoretical changes (entries of the character table) may not be trivial and should be worked out and evaluated by experts. The basic group-theoretical approach [1] was the creation of a group $G$ of order 240 by linking the finite group $Z_{5}$ of order 5 with the binary octahedral $2 O$ group of order 48 .

\section{Helical Structures}

Helical structures dominate or life in the form of the $\alpha$-helix of proteins [9] [10] [11] or double-helices composed of double-stranded molecule chains of nucleic acids such as DNA [10]. Helical curling of cellulosic micro-fibrils leads to spring-like elastic behavior [12]. Interesting is that helical left-handedness and its opposite can be realized, when a single cellulosic fiber is supported at both ends [13]. Designers have many learnt from the multilayer tube-like structure of wood cell walls leading to superior properties of this natural material [14]. Let's stick with technical aspects such as making torque from wind. The wake behind multi-bladed rotors can be described by interlaced helical vortices [15]. But also liquid water forms helices of pentagonal or hexagonal symmetry [16]. When subtracting from the regular tetrahedron angle of $109.47^{\circ}$ the angle $\arcsin \left(\varphi^{5}\right)=5.17^{\circ}$, where $\varphi$ is the golden mean, then the bonding angle of $\mathrm{H}_{2} \mathrm{O}$ is quite well adapted giving $104.30^{\circ}$. This connection to the golden mean hierarchy is intriguing. It is reasonable to assume that water may be involved in two ways in the origin of life, as a liquid medium and as helical scaffold mediating the first assembling of a double-helix of nucleic acids. In contrast, the participation of structurally disordered clay minerals in this process is very unlikely. The fifths power of the golden mean $\varphi^{5}$ in turn is frequently connected to phase transformation from microscopic to cosmic scale [4] [17]. This number may be characterized as a universal one [18]. On the border between hexagonal and pentagonal respectively Fibonacci number governed symmetry our existence is located. Numerical it may be emphasized by the relation $\varphi^{5}=5 \varphi-3$.

Turning to inorganic structures, in 1977 I solved the hexagonal palatinate crystal structure of $\mathrm{Cs}_{2}\left[\mathrm{Pt}(\mathrm{CN})_{4}\right] \cdot \mathrm{H}_{2} \mathrm{O}$, in which planar $\mathrm{Pt}(\mathrm{CN})_{4}$ groups are helical winding down the $c$ axis by means of a $6_{1}$ screw axis (see Figure 2) [19]. It represents a nice example of helicity in inorganic matter. At that time, I have discussed the $\mathrm{Pt}$ orbital overlap of this interesting inorganic compound with 
Nobel laureate Linus Pauling who solved the structure of the $\alpha$-helix of proteins 26 years before [9] [10] [11].

Since that time I was fascinated by the helical structure of matter, but found no time to think about it anymore considering crystal-chemical, biochemical as well as mathematical aspects.

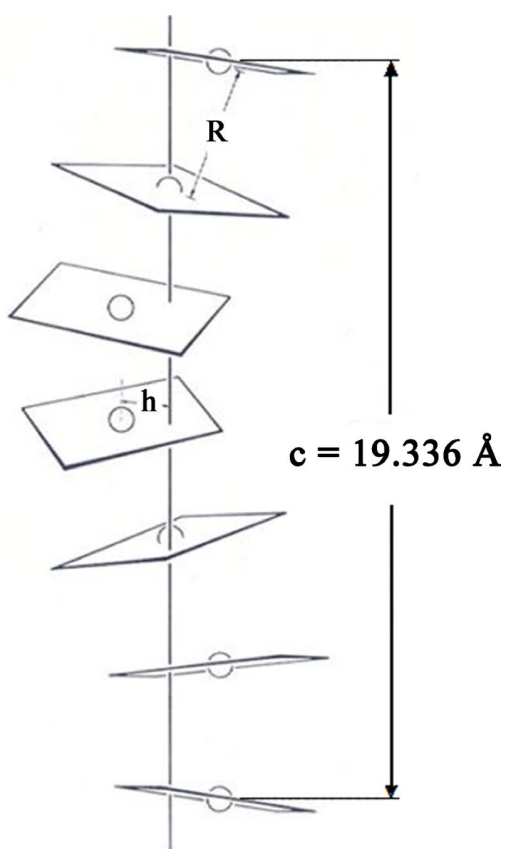

Figure 2. Helix of $\mathrm{Pt}(\mathrm{CN})_{4}$ squares screwed down the $c$ axis of $\mathrm{Cs}_{2}\left[\mathrm{Pt}(\mathrm{CN})_{4}\right] \cdot \mathrm{H}_{2} \mathrm{O}$ [19]. $R$ $=\mathrm{Pt}$ atomic distance, $h=\mathrm{Pt}$ distance from the [001] origin line.

\section{A Number-Theoretical Golden Helix Gimmick}

Now we turn to the $\alpha$-helix peculiarities of proteins where 18 subunits perform 5 turns to reach an identical position when projected down the fiber axis [9] [10]. This means one needs 3.6 subunits per turn. This number is nearby another golden mean derived number (see Equation (2))

$$
3+\varphi=3.6180339887 \cdots=\sqrt{13+\varphi^{5}}=\sqrt{2+\varphi^{-5}}
$$

In case of the $\alpha$-helix, when tentatively using $3.61803 \ldots$ subunits per turn instead of 3.6, one would end with $18+\varphi^{5}$ subunits after 5 turns

$$
5\left(2+\varphi^{-1}\right)=\left(\varphi^{5}+\varphi^{-5}\right) \varphi^{-1}=18+\varphi^{5}=18.0901699 \cdots
$$

and again the fifth power of the golden mean would be involved.

What marginal change would such approach of helix breathing produce in the Fourier transform possibly overseen as yet by the interpretation of the diffraction patterns showing quite broad smeared out diffraction spots? An elaborated study of structural variations of $B-D N A$ by interpreting features of its diffraction pattern was recently given in [20].

The value of $\sqrt{3+\varphi}=\sqrt{1+\varphi^{-2}}$ is also related to an icosahedron as its circumradius when this polyhedron with an edge length of $a=2$ is centered at the 
origin [5] [21]. It may be considered as a very important distance frequently found in the following reflections.

Turning to five-fold symmetrical nucleic acid structures discussed in [1], the regular pentagon with side length $a$ has an area of $A_{5}$ calculated by [21]

$$
A_{5}=\frac{5}{4 \varphi^{2} \sqrt{3+\varphi}} a^{2}=1.720477403 a^{2}
$$

Another example should be considered with respect to the seven-fold symmetry of complexes like the Lsm1-7 one recently analyzed by Planat et al. [22] [23]. The geometry of a heptagon delivers a connection to the golden mean. The heptagon area $A_{7}$ with side length a yields also about $\sqrt{3+\varphi}$ in its expression

$$
\begin{gathered}
A_{7}=\frac{7}{4 \cdot \tan \left(\frac{180}{7}\right)} a^{2}=3.633912 a^{2} \approx(3+\varphi) a^{2}=3.618033987 a^{2} \\
\quad \text { and } \tan \left(\frac{180}{7}\right)=0.481574618 \approx \ln \left(\varphi^{-1}\right)=0.481211826
\end{gathered}
$$

The relative area difference between heptagon and pentagon can be approximated by

$$
\frac{A_{7}-A_{5}}{A_{5}}=1.11169295 \approx \sqrt{1+\varphi^{3}}=1.1117859
$$

Another example from number theory where the golden mean in form of the square root term $\sqrt{3+\varphi}$ is involved but not yet expected is the series of heptagonal numbers generated when heptagons with ascending size are combined [24]. Such figurative heptagonal numbers are generated by the formula [24]

$$
P_{n}=\frac{5 n^{2}-3 n}{2}=1,7,18,34,55,81, \cdots
$$

indicating a parity sequence of odd, odd, even, even ... and successive Lucas numbers 7 and 18 [25] respectively Fibonacci numbers 34 and 55 [26].

The sum of reciprocals of this number series shows a result in full glory, if we recast for the first time the already known expression [24] using the golden mean as $\varphi=\frac{\sqrt{5}-1}{2}$

$$
\begin{aligned}
\sum_{n=1}^{\infty} \frac{1}{P_{n}} & =\frac{2}{3}\left(\frac{\pi}{2} \frac{\varphi}{\sqrt{1+\varphi^{-2}}}+\ln (5)+\varphi^{-1} \ln \left(\sqrt{1+\varphi^{2}}\right)-\varphi \cdot \ln \left(\sqrt{1+\varphi^{-2}}\right)\right) \\
& =1.3227792531 \cdots \approx \frac{2}{3} \ln (5)+\frac{1}{4}
\end{aligned}
$$

Alternatively, it applies because $\sqrt{1+\varphi^{2}}=\varphi \sqrt{1+\varphi^{-2}}=\varphi \sqrt{3+\varphi}$

$$
\begin{aligned}
\sum_{n=1}^{\infty} \frac{1}{P_{n}} & =\frac{2}{3}\left(\frac{\pi}{2} \frac{\varphi}{\sqrt{3+\varphi}}+\ln (5)+\frac{\ln (\varphi)}{\varphi}+\ln (\sqrt{3+\varphi})\right) \\
& =\frac{2}{3}\left(\frac{\pi}{2} \frac{\varphi}{\sqrt{3+\varphi}}+\ln \left(5 \cdot \varphi^{1 / \varphi}\right)+\ln (\sqrt{3+\varphi})\right)
\end{aligned}
$$


where

$$
\frac{\ln (\sqrt{3+\varphi})}{\ln \left(5 \cdot \varphi^{1 / \varphi}\right)}=2.289438326 \approx 5 \cdot \varphi^{1 / \varphi}=2.29520192
$$

and further

$$
\frac{1}{2} \frac{\sqrt{3+\varphi}}{5 \cdot \varphi^{1 / \varphi}}=0.414367253 \approx(\sqrt{2}-1)=0.414213562
$$

$\sqrt{2}-1$ represents the silver mean here approximated by an expression for the golden mean.

It indicates that even in the case of seven-fold symmetry a sophisticated numerical relationship to $\varphi$, the omnipresent golden code number of life, is given and should be considered in future work on the genetic code and resonance behavior of life. The relation between $\sqrt{3+\varphi}$ respectively $\varphi^{5}$ with the circle number $\pi$ is also remarkable (see also Appendix)

$$
\begin{gathered}
\sqrt{3+\varphi}=1.902113 \approx \frac{6}{\pi}=1.909859 \\
\varphi^{5}=0.0901699 \approx 2-\frac{6}{\pi}=0.0901406
\end{gathered}
$$

\section{Simple Didactic Approach for the Fourier Transform of a Helix}

The Fourier transform of a helical structure resulting in the integral representation of Bessel functions of different order was first introduced in 1952 by Cochran [9] and can be studied in detail in the monograph "Direct Analysis of Diffraction by Matter" by Hosemann and Bagchi [27]. Another recent contribution by Latychevskaia and Fink [28] is recommended as well as that given by Strutz in German [29].

Our simplified but didactic approach is as follows. If one looks through the projection of a helical structure perpendicular to the filament direction, one will see the mirror image on the back at the helix diameter distance to the front image, somewhat offset in filament direction depending on the helix winding parameters. One can perform the Fourier summation to obtain diffraction intensities exemplified by the projected hexagonal nets of a special Fibonacci micro-tubule with a 13 steps full turn. The starting small cell is helically wound at the twist angle $\alpha$. It needs 13-times the small cell lattice parameter $a_{\text {sub }}$ to reach again identity with a large cell lattice point. In this way, the diameter of the smallest geometrically possible microtubule is calculated to be $d_{1} \approx 13 a_{\text {sub }} \cdot \cos (\alpha) / \pi=3.5 \cdot a / \pi$. Interesting is $d_{6}=21 \cdot \frac{a}{\pi}$ showing a further Fibonnaci number. By a full turn one gains a height in filament direction of

$$
h_{1}=\frac{\sqrt{39}}{2} a_{\text {sub }}=\frac{\sqrt{3}}{2} \sqrt{13} \cdot a_{\text {sub }}=3.1225 \cdot a_{\text {sub }} \approx \pi \cdot a_{\text {sub }} .
$$


More detail of the chosen example can be found in [30] [31]. However, when again choosing in Equation (13) a value of $\sqrt{13+\varphi^{5}}$ instead of $\sqrt{13}$ by a small change of the twist, then $\frac{h_{1}}{a_{\text {sub }}}=3.1333$ would better approximate $\pi$ regardless whether this can be significant in any way.

The example simplifies helically twisted tubulin microtubules that also show the strongest reinforcement of the ordered pattern when the proto-filament number $n$ equals 13 [32] [33]. Interestingly, according to Penrose and Hamedoff tubulin microtubules are considered as the locus where information is stored and assessed, what constitutes our consciousness and cognition [34] [35]. This supports the proposal for a twisted Fibonnaci net as displayed in Figure 3 respectively Figure 4 as a quantum computer bio-template.

Information may be preserved in tubulin molecules by structural adaption and sequence fluctuations disrupting the helical coherence.

When removing from the Fibonacci net some atomic stripes one can simulate a double helix. Detailed intensity calculation of such a construct will be given in a forthcoming contribution [7] showing the characteristic $X$ shaped cross of diffraction intensity distribution of a double helix similar to the electron diffraction pattern of B-DNA fibers [36] respectively to the historical $X$-ray pattern (photo 51) of B-DNA taken by the felt Nobel laureate of hearts Rosalind Franklin [37]. When one works with the projected curved net instead of a flat net, the reflections smear out as result of the introduced coordinate statistics. Figure 3 may
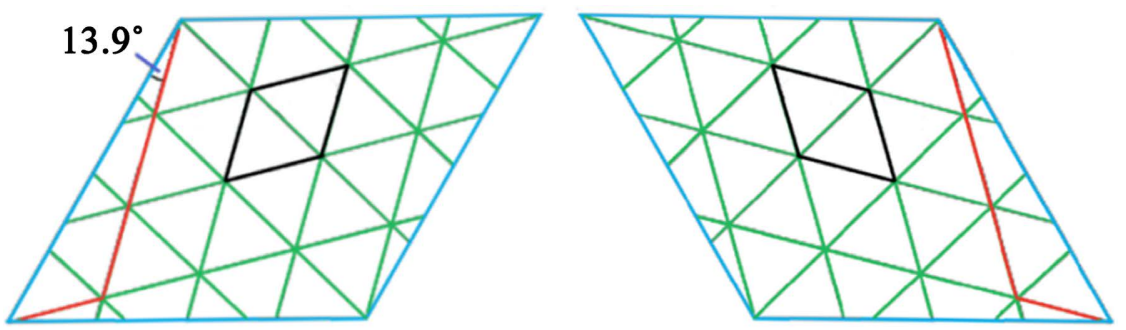

Figure 3. Fibonacci arrangement of a hexagonal net and its mirror image [4] [29] [30]. The light-blue outlined unit-cell contains 13 sub-cells, offset by an angle of $\alpha=13.9^{\circ}$.

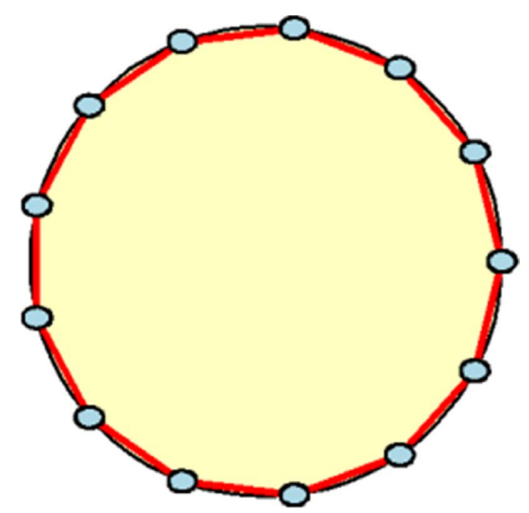

Figure 4. Helically twisted Fibonacci microtubule projected down the filament direction with 13 light-blue atoms or atom groups on sub-lattice positions. 
also help to understand in which way inverted repeat of nucleotides works capable of self-complementary base pairing.

\section{Beyond the Icosahedron Equation}

If the genetic information is ultimately coiled and compacted into an icosahedral shape as found in viruses, then another function may come into play, represented by the icosahedron equation mapping for instance the positions of the face centers of an icosahedron with unit in-radius projected onto a complex plane where $Z$ is the coordinates [38] [39] [40] [41]

$$
H(z, 1)=z^{20}-228 z^{15}+494 z^{10}+228 z^{5}+1
$$

The roots accordingly correspond to the locations of the face midpoints on the Riemann sphere. Interestingly but not surprisingly, the coefficient ratio yields $494 / 228=13 / 6$ and contains the Fibonacci numerator 13. The coefficients 228 as well as 494 can be replaced by a golden mean based approximation besides number 13

$$
\begin{gathered}
228 \approx \frac{4}{3}\left(13+\frac{\varphi^{4}}{\sqrt{3+\varphi}}\right)^{2}=228.00022 \\
228 \approx \frac{4}{3}\left(13+\frac{1}{13}\right)^{2}=228.00789 \\
494 \approx \frac{2}{9} \cdot 13\left(13+\frac{1}{13}\right)^{2}=494.01709
\end{gathered}
$$

Based on the preliminary work by Gordon [42], Klein showed the connection between the regular icosahedron, one of the five Platonic bodies, and the solution of the quintic polynomial. Instead of following Klein's quintic icosahedral solution, the substitution of the complex variable $z^{5} \rightarrow x$ formally leads to a quartic polynomial

$$
\begin{aligned}
H(x, 1) & =x^{4}-228 x^{3}+494 x^{2}+228 x+1 \\
& \approx x^{4}-\frac{4}{3}\left(13+\frac{1}{13}\right)^{2} x\left(x^{2}-\frac{13}{6} x-1\right)+1
\end{aligned}
$$

The four root of this polynomial have been calculated giving

$$
\begin{gathered}
x_{3}=-\frac{1}{x_{2}}, x_{4}=-\frac{1}{x_{1}} \\
x_{3}=2.58365 \approx 228-\frac{4}{3} 13^{2}=2.66666 \\
x_{4}=225.80782 \approx \frac{4}{3} 13^{2}=225.33333
\end{gathered}
$$

In addition, it yields

$$
\sum_{i=1}^{4} x_{i}=228
$$

The reader is frequently confronted with the Fibonacci number 13, which ob- 
viously plays an important role besides $\varphi$ and $\varphi^{5}$ when assessing bio-coding and related storage and processing of information.

Turning to another polynomial equation corresponding to the vertices of an icosahedron [41]

$$
w(x)=x\left(x^{10}+11 x^{5}-1\right)
$$

Now again substituting $z$ for $x^{5}$ in the term in brackets and solving the resulting quadratic equation (only bracket term)

$$
z^{2}+11 z-1=0
$$

where

$$
11=\varphi^{-5}-\varphi^{5}
$$

one can confirm the two roots as $z_{1}=\varphi^{5}$ respectively $z_{2}=-\varphi^{-5} \quad$ (see also [4]).

In a recent contribution Planat et al. [23] defined a model for the structure of a nucleosome as secondary unit consisting of 8 histone proteins with a $D N A$ double helix wound around. The assigned group was $\boldsymbol{G}_{8}=(8 \cdot 48 ; 5589) \cong \boldsymbol{Z}_{8} \rtimes 2 \boldsymbol{O}$, where the finite group $Z_{8}$ is of order 8 , the binary octahedral group $2 O$ is of order 48 , and $d=37$ is the dimension of the corresponding Pauli group. One can approximate the rank of the Gram matrix $d^{2}=1369$ and the entries of 1367 in the structure table 5 [23]) by

$$
\begin{aligned}
& 8 \cdot\left(13+\frac{1}{13}\right)^{2}=1368.047 \approx 1368 \\
& 8 \cdot\left(13+\frac{\varphi^{4}}{\sqrt{3+\varphi}}\right)^{2}=1368.0013
\end{aligned}
$$

Again one has a result showing the before derived square term based on number 13 (see Equation (21)). Furthermore, relating the coefficients of Equation (19) showing number 1368 one confirms simply $228 \cdot 6=1368$ respectively $494 \cdot \frac{6^{2}}{13}=1368$.

\section{Self-Duality as a Suggested Property for Image Storage, Memory and Consciousness}

Self-duality is a very important concept of mathematics as well as physics and should be applied to biophysics respectively biochemistry in order to understand the very stable information storage within the human brain. Self-dual objects include polyhedra, graphs, configurations and codes. The skeleton of a self-dual polyhedron is a self-dual graph. For instance, self-dual solitons as self-reinforcing and shape-maintaining wave packets are expected to be very stable. Among the self-dual polyhedra are the regular tetrahedron respectively pyramids in general. Also the Kummer quartic surface with 16 nodes and 16 singular tangent conics, believed by Planat et al. [22] [23] to explain in part the genetic code of secondary protein structures besides $D N A$, is self-dual [43]. 
The information storage respectively processing certainly doesn't work via simple bits or qubits but via complex pictures generated by superposition of stable (soliton-like) wave packets, burned into a multiplex microtubule array or a comparable matrix. This happens against the background of the omnipresent dark energy with all the information collected up to then. Each speedy moving body or particle is surrounded by dark matter respectively energy [17], and when seeking for an assumed resonance field that links $D N A$ or tubulin resonances with the work of the brain according to [6], the first candidate to fulfill all conditions is simply dark energy.

\section{What Is the Code for an Assumed Electron Helix?}

Importantly, also elementary particles such as the electron are thought to exhibit a helical structure [44] [45] indicating that nature used this principle of curling again and again [46]. Remarkably, the arithmetic mean between the proposed maximal superluminal electron velocity and the minimal one yields $\bar{v}=\frac{c}{\varphi}$. The application of IRT transformations [17] to the problem would certainly present the electron helix approach correctly and in full glory. We will work on this. The frequency of electron's Zitterbewegung is found to equal that of the composing photons [44]. Besides the results given in [47] [48] [49] and contrasting the QED coding that uses an unbelievable cascade of Feynman diagrams [50] [51] [52] one should try to encode and understand a numerical golden mean relation for the electron as a quite stable helically wound and less spherically than icosahedron-shaped compacted entity where $\Delta g_{e}$ is the gyromagnetic correction factor of the electron. Using just derived algebraic-geometrical relations, one can our fully golden mean based approximation of $\Delta g_{e}$ [47] [48] equip with a more structured meaning pointing to a possible virus-like structure of the electron

$$
\Delta g_{e} \approx \frac{\varphi^{6}}{24} \approx \frac{5}{3} \cdot \frac{\sqrt{3+\varphi}}{8\left(13+\frac{1}{13}\right)^{2}}=0.0023174
$$

respectively

$$
\Delta g_{e} \approx \frac{\varphi-\exp (-\varphi)}{34+\varphi^{5}}=0.00231829
$$

The experimental value of $\Delta g_{e}$ was determined slightly larger [51] [52], but a new relativistic correction was proposed shifting this value towards the considered smaller one [48].

If we proceed with this helix quantum-vortex approach we may at least understand electron's pairing in superconductors, too [53]. Historically, already in 1978 L. S. Levitt had raised the question whether the photon is a double helix [54]. A photon with high energy decomposes by interaction with matter to an electron and a positron, both helically twisted. Recently, Williamson suggested a 
possible structure of the spherical electron (positron) by an elaborated new linear theory of light and matter [55], but used once again the questionable relativistic factor $\gamma=\frac{1}{2}\left(R+\frac{1}{R}\right)$, where the Doppler factor is given as $=\sqrt{\frac{1+\beta}{1-\beta}}$, and $\beta=\frac{v}{c}$.

However, if one could ultimately prove the double helix structure of the electron, it would seem natural to think further of its possible fine structure making one electron not equal to another one when interacting with the environment, despite their same mass and bulk properties. It would revolutionize our ideas when it comes to storing and processing exorbitantly large amounts of information.

\section{Helical Helix Construct}

Single respectively double-strand helices may not only be found in nature as rotational blurred linearly aligned fibers. The fiber axis of a helix or double-helix may itself be proposed in turn helically wound to strengthen its spring-like elastic behavior suggested for application in building constructions. Such construct would not represent the densest packing, but an extremely flexible one. I am not sure whether nature used this construct, for instance as $D N A$ partial packing in viruses or in the structure of the electron, but designers could certainly gain profit in its application. Also energy harvesting may be possible when such construct was set in vibration by wind or water.

\section{Conclusion}

The given suggestions are intended to provide an expanded view on the already complicated matter of coding important helical structures of life and matter exemplified by the DNA or the beautiful electron. The duality of matter and surrounding waves or matter and dark matter leaves fingerprints in such structures in form of numerical reciprocity given by the golden mean $\varphi^{-1}=\varphi+1=\varnothing$, besides $\varphi^{-5}+\varphi^{5}=11$ (phase transitions from microscopic to cosmic scale, dimension of superstring theory) or $\frac{2}{3}\left(13+\frac{1}{13}\right)^{2} \approx 228$ (coefficient of the icosahedron equation). Number 13 is also found as proto-filament number in helically twisted tubulin microtubules as the locus where human information storage and assessment is suspected. Philosophically one could say that life beats 13 . The reciprocity symmetry principles may find application in topological quantum computers based on Fibonacci anyons simulating processes that determine the speed and storage capacity of human mind.

\section{Acknowledgements}

Many colleagues inspired this contribution, focussing all the years on the importance and beauty of the golden mean or golden ratio present in all areas of 
life, physics and the entire cosmos. Especially the author gratefully acknowledges the stimulating work of El Naschie, Scott Olsen, Leila Marek-Crnjac, Ji-Huan He, and Michael Sherbon.

\section{Conflicts of Interest}

The author declares no conflicts of interest regarding the publication of this paper.

\section{References}

[1] Planat, M., Aschheim, R.A., Amaral, M.M., Fang, F. and Irwin, K. (2020) Complete Quantum Information in the DNA Genetic Code. Symmetry, 12, 1993. https://doi.org/10.3390/sym12121993

[2] Hardy, L. (1993) Nonlocality for Two Particles without Inequalities for Almost All Entangled States. Physical Review Letters, 71, 1665-1668. https://doi.org/10.1103/PhysRevLett.71.1665

[3] Mermin, N.D. (1994) Quantum Mysteries Refined. American Journal of Physics, 62, 880-887. https://doi.org/10.1119/1.17733

[4] Otto, H.H. (2020) Phase Transitions Governed by the Fifth Power of the Golden Mean and Beyond. World Journal of Condensed Matter Physics, 10, 1-22. https://doi.org/10.4236/wjcmp.2020.103009

[5] Otto, H.H. (2020) Magic Numbers of the Great Pyramid: A Surprising Result. Journal of Applied Physics and Mathematics, 8, 2063-2071. https://doi.org/10.4236/jamp.2020.810154

[6] Savelyev, I., Polesskaya, O., Zyryanova, N. and Myakishev-Rempel, M. (2019) On the Existence of The DNA Resonance Code and Its Possible Mechanistic Connection to The Neural Code. NeuroQuantology, 17, 56-71. https://doi.org/10.14704/nq.2019.17.2.1973

[7] Otto, H.H. (2021) Generalization of Helical Structures in Inorganic Matter and Nature.

[8] Tehrani, F.T. (2020) Solution to Polynomial Equations, a New Approach. Applied Mathematics, 11, 53-66. https://doi.org/10.4236/am.2020.112006

[9] Pauling, L., Corey, R.B. and Bronson, H.R. (1951) The Structure of Proteins; Two Hydrogen-Bonded Helical Configurations of the Polypeptide Chain. Proceedings of the Academy of Science, 37, 205-211. https://doi.org/10.1073/pnas.37.4.205

[10] Cochran, W., Crick, F.H.C. and Vand, V. (1952) The Structure of Synthetic Polypeptides. I. The Transform of Atoms on a Helix. Acta Crystallographica, 5, 581-586. https://doi.org/10.1107/S0365110X52001635

[11] Pauling, L. (1960) Chemie-Eine Einführung. Verlag Chemie GmbH, Weinstein, 516-526.

[12] Watson, J.D. and Crick, F.H.C. (1953) Molecular Structure of Nucleic Acids. A Structure for Deoxyribose Nucleic acid. Nature, 171, 737-738. https://doi.org/10.1038/171737a0

[13] Godinho, M.H., Canejo, J.P., Feio, G. and Terenjev, E.M. (2010) Self-Winding of Helices in Plant Dendrils and Cellulose Liquid Crystalline Fibers. Soft Matter, 6, 5965-5970. https://doi.org/10.1039/c0sm00427h

[14] Zoretti, L. and Ruffoni, D. (2018) Wood-Inspired 3D-Printed Helical Composites with Tunable and Enhanced Mechanical Performance. Advanced Functional Materials, 
29, Article ID: 1805888. https://doi.org/10.1002/adfm.201805888

[15] Quantara, H.U., Brynjell-Rahkola, M., Leweke, T. and Henningson, D.S. (2016) Long-Wave Instabilities of Two Interlaced Helical Vortices. Journal of Physics: Conference Series, 753, Article ID: 032022. https://doi.org/10.1088/1742-6596/753/3/032022

[16] Lozynski, M. (2015) Liquid Water: The Helical Perspective of Structure. Chemical Physics, 455, 1-6. https://doi.org/10.1016/j.chemphys.2015.03.013

[17] Suleiman, R. (2019) Relativizing Newton. Nova Scientific Publisher, New York, 1-207.

[18] Otto, H.H. (2020) A Primer of Important Natural Numbers and Revisited Fundamental Physical Constants. Researchgate.net, 1-4.

[19] Otto, H.H., Schulz, H., Thiemann, K.H., Yersin, H. and Gliemann, G. (1977) Crystal Structure of $\mathrm{Cs}_{2}\left[\mathrm{Pt}(\mathrm{CN})_{4}\right] \cdot \mathrm{H}_{2} \mathrm{O}$. Zeitschrift für Naturforschung, 32b, 127-130. https://doi.org/10.1515/znb-1977-0203

[20] Kornyshev, A.A., Lee, D.J., Wynveen, A. and Leikin, S. (2011) Signatures of DNA Flexibility, Interaction and Sequences-Related Structural Variations in Classical X-Ray Diffraction Patterns. Nucleic Acids Research, 39, 7289-7299. https://doi.org/10.1093/nar/gkr260

[21] Otto, H.H. (2021) Ratio of In-Sphere Volume to Polyhedron Volume of the Great Pyramid Compared to Selected Convex Polyhedral Solids. Journal of Applied Physics and Mathematics, 9, 41-56. https://doi.org/10.4236/jamp.2021.91005

[22] Planat, M., Chester, D., Aschheim, R., Amaral, M.M., Fang, F. and Irwin, K. (2021) Finite Groups for the Kummer Surface: The Genetic Code and a Quantum Gravity Analogy. Quantum Reports, 3, 68-79. https://doi.org/10.3390/quantum3010005

[23] Planat, M., Aschheim, R., Amaral, M.M., Fang, F. and Irwin, K. (2021) Quantum Information in the Protein Codes, 3-Manifolds and the Kummer Surface. Symmetry, 13, 1146. https://doi.org/10.3390/sym13071146

[24] Heptagon. https://en.wikipedia.org/wiki/Heptagonal

[25] Lucas, E. (1891) Theorie des nombres. Gauthier-Villars, Paris.

[26] Pisano, L. (1202) Fibonacci's Liber Abaci (Book of Calculation). Biblioteca a Nazionale di Firenze.

[27] Hosemann, R. and Bagchi, S.N. (1962) Direct Analysis of Diffraction by Matter. North-Holland Pubishing Company, Amsterdam.

[28] Latychevskaia, T. and Fink, H.W. (2018) Three-Dimensional Double Helical DNA Structure Directly Revealed from Its X-Ray Fiber Diffraction Pattern by Iterative Phase Retrieval. Optics Express, 28, 30991-31017. https://doi.org/10.1364/OE.26.030991

[29] Strutz, Ch. (2003) Bessel-Funktionen für Röntgen-Strukturanalyse der Alpha-Helix und der Doppelhelix. Researchgate.net, 1-7.

[30] Otto, H.H. (2015) Pyroelectric $\mathrm{Bi}_{5-\mathrm{x}}\left(\mathrm{Bi}_{2} \mathrm{~S}_{3}\right)_{39} \mathrm{I}_{12} \mathrm{~S}$ : Fibonacci Superstructure, Synthesis Options and Solar Cell Potential. World Journal of Condensed Matter Physics, 5, 66-77. https://doi.org/10.4236/wjcmp.2015.52010

[31] Otto, H.H. (1965) Zur Kristallchemie synthetischer Blei-Wismut-Spießglanze. Thesis, TU, Berlin.

[32] Wade, R.H., Chrétien, D. and Job, D. (1990) Characterization of Microtubule Protofilament Numbers. How Does the Surface Lattice Accommodate? Journal of Molecular Biology, 212, 775-786. https://doi.org/10.1016/0022-2836(90)90236-F 
[33] Ti, S.C., Alushin, G.M. and Kapoor, T.M. (2018) Human ß-Tubulin Isotypes Can Regulate Microtubule Protofilament Number and Stability. Developmental Cell, 47, 175-190. https://doi.org/10.1016/j.devcel.2018.08.014

[34] Penrose, R. and Hamedoff, S.R. (1995) Quantum Computing in Microtubules: Self-Collapse as a Possible Mechanism for Consciousness. Toward a Science of Consciousness. MIT Press, Cambridge.

[35] Penrose, R. and Hamedoff, S.R. (2011) Consciousness in the Universe: Neuroscience, Quantum Space-Time Geometry and Orch or Theory. Journal of Cosmology, 14, 1-50.

[36] Gentile, F., et al. (2012) Direct Imaging of DNA Fibers: The Visage of Double Helix. Nanoletters, 12, 6453-6458. https://doi.org/10.1021/nl3039162

[37] Franklin, R. and Gosling, R.G. (1953) Molecular Configuration in Sodium Thymonucleate. Nature, 171, 740-741. https://doi.org/10.1038/171740a0

[38] Klein, F. (1884) Vorlesungen über das Ikosaeder und die Auflösung der Gleichungen vom fünften Grad. Verlag B. G. Teubner, Leipzig.

[39] Slodowy, P. (1986) Das Ikosaeder und die Gleichungen fünften Grades. Arithmetik und Geometrie. Mathematische Miniaturen, 3, 71-113. https://doi.org/10.1007/978-3-0348-5226-5_3

[40] Eschenburg, J.H. (1997) Das Ikosaeder und die Gleichungen 5. Grades nach Felix Klein. Seminar am Mathematischen Institut der Universität Augsburg, 8Nov. 1997/ Feb. !999), 1-29.

[41] Nash, O. (2013) On Klein's Icosahedral Solution of the Quintic. Expositiones Mathematicae, 32, 99-120. https://doi.org/10.1016/j.exmath.2013.09.003

[42] Gordon, P. (1869) Über die Auflösung der Gleichung vom fünften Grade. Mathematische Annalen, 13, 375-404. https://doi.org/10.1007/BF01447396

[43] Dolgachev, I. (2020) Kummer Sarfaces: 200 Years of Study. Notices of the American Mathematical Society, 67, 1527-1533. https://doi.org/10.1090/noti2168

[44] Gauthier, R. (2013) Transluminal Energy Quantum Models of the Photon and the Electron. In: The Physics of Realty. Space, Time, Matter, Cosmos, World Scientific, Hackensack, 445-452. https://doi.org/10.1142/9789814504782_0045

[45] Gauthier, R. (2019) Quantum-Entangled Superluminal Double-Helix Photon Produces a Relativistic Superluminal Quantum-Vortex Zitterbewegung Electron and Positron. IOP Conference Series. Journal of Physics: Conference Series, 1251, Article ID: 012016. https://doi.org/10.1088/1742-6596/1251/1/012016

[46] Otto, H.H. (2017) Should We Pay More Attention to the Relationship between the Golden Mean and the Archimedes' Constant? Nonlinear Science Letters A, 8, 410-412.

[47] Otto, H.H. (2017) Gyromagnetic Factor of the Free Electron: Quantum-ElectroDynamical Correction Expressed Solely by the Golden Mean. Nonlinear Science Letters $A, \mathbf{8}, 413-415$.

[48] Otto, H.H. (2020) Reciprocity as an Ever-Present Dual Property of Everything. Journal of Modern Physics, 11, 98-121. https://doi.org/10.4236/jmp.2020.111007

[49] Gabrielse, G., Hanneke, D., Kinoshita, T., Nio, M. and Odom, B. (2007) Erratum: New Determination of the Fine Structure Constant from the Electron $g$ Value and QED. Physical Review Letters, 99, Article ID: 039902. https://doi.org/10.1103/PhysRevLett.99.039902

[50] Gabrielse, G., Hanneke, D., Kinoshita, T., Nio, M. and Odom, B. (2006) New Determination of the Fine Structure Constant from Electron g Value and QED. Physi- 
cal Review Letters, 97, Article ID: 030802. https://doi.org/10.1103/PhysRevLett.97.030802

[51] Odom, B. (2004) Fully Quantum Measurement of the Electron Magnetic Momentum. Thesis, Harvard University, Cambridge.

[52] The NIST Reference of Constants, Units and Uncertainty, NIST Gaitherburg, MD 20899, USA.

[53] Otto, H.H. (2016) A Different Approach to High- $\mathrm{T}_{\mathrm{c}}$ Superconductivity: Indication of Filamentary-Chaotic Conductance and Possible Routes to Superconductivity above Room Temperature. World Journal of Condensed Matter Physics, 6, 244-260. https://doi.org/10.4236/wjcmp.2016.63023

[54] Levitt, L.S. (1978) Is the Photon a Double Helix? Lettere al Nuovo Cimento, 21, 222-223. https://doi.org/10.1007/BF02822254

[55] Williamson, J.G. (2019) A New Linear Theory of Light and Matter. Journal of Physics. Conference Series, 1251, Article ID: 012050.

https://doi.org/10.1088/1742-6596/1251/1/012050

[56] Olsen, S. (2006) The Golden Section: Nature's Greatest Secret. Bloomsbury, 64 p.

[57] Sherbon, M.A. (2014) Fundamental Nature of the Fine-Structure Constant. International Journal of Physical Research, 2, 1-9.

https://doi.org/10.14419/ijpr.v2i1.1817

[58] Otto, H.H. (2017) Continued Fraction Representation of Universal Numbers and Approximations. Researchgate.net, 1-7.

[59] El Naschie, M.S. (2017) Elements of a New Set Theory Based Quantum Mechanics with Application in High Quantum Physics and Cosmology. International Journal of High Energy Physics, 4, 65-74. https://doi.org/10.11648/j.ijhep.20170406.11

[60] Jelic, V. and Marsiglio, F. (2012) The Double Well Potential in Quantum Mechanics: A Simple Numerical Exact Formulation. European Journal of Physics, 33, 1651. https://doi.org/10.1088/0143-0807/33/6/1651

[61] Suleiman, R. (2020) Beauty in Motion through the Lens of Doppler's Formula. 1-23. https://vixra.org/abs/2010.0048 


\section{Appendix}

\section{Golden Mean Beauty and Its Intrinsic Reciprocity Property [48]}

The golden mean or golden ratio $\varphi$ is an omnipresent number in nature, found in the architecture of living creatures as well as human buildings, music, finance, medicine, philosophy, and of course in physics and mathematics [56] [57]. It is the most irrational number known and a number-theoretical chameleon with a self-similarity property. On the other hand, its infinite continued fraction representation is the simplest of all and is represented together with that for its fifth power by [58]

$$
\varphi=\frac{\sqrt{5}-1}{2}=\frac{1}{1+\frac{1}{1+\frac{1}{1+\cdots}}}, \quad \varphi^{5}=\frac{1}{11+\frac{1}{11+\frac{1}{11+\cdots}}}
$$

It impressively underlines the fractal character of both universal numbers. Most obviously, the golden mean mediates stability of a system, because only "particles" as the center of gravity of vibrations with the most irrational winding survive. Important numerical relations involving $\varphi$ are summarized below. However, to prevent confusion, in textbooks of mathematics the reciprocal value for $\varphi$ is frequently used.

$$
\begin{gathered}
\varphi=\frac{\sqrt{5}-1}{2}=0.618033988 \cdots, \quad \varphi^{-1}=1+\varphi=\frac{\sqrt{5}+1}{2}=1.618033988 \cdots \\
\varphi^{2}=1-\varphi=0.381966011 \cdots, \quad \varphi^{-2}=2+\varphi=2.618033988 \cdots \\
\left(\varphi^{2}+1\right)^{-1}=\left(\varphi^{-2}+1\right) / 2
\end{gathered}
$$

or equivalently

$$
\begin{gathered}
\left(\varphi^{-2}+1\right)^{-1}=\left(\varphi^{2}+1\right) / 5 \\
\varphi^{5}=\frac{1-\varphi}{1+\varphi} \varphi^{2}=0.090169943 \cdots \quad[2] \\
\frac{\varphi^{5}}{2}+\frac{5}{2} \varphi^{2}=1 \\
\varphi^{3}+2 \varphi^{2}=1
\end{gathered}
$$

Connecting the fifth power of $\varphi$ with the circle constant $\pi$ on can confirm

$$
\pi \cdot \varphi^{5}=0.283277 \approx 2 \pi-6=0.283185
$$

and further

$$
\varphi^{3} \approx \frac{5}{3}(\pi-3)
$$

The product of these fundamental numbers is found as volume quotient of the in-sphere of the Great Pyramid to the pyramid volume itself [5] [21].

Solution of a Special Quadric Polynomial Equation

The approach $\frac{4}{3}\left(x+x^{-1}\right)^{2}=228$ of Equation (21) can be recast into the qu- 
adric polynomial equation

$$
x^{4}-169 x^{2}+1=0
$$

with solutions $x_{1,2}= \pm 12.99977241 \approx \pm 13$ and $x_{3,4}=x_{1,2}{ }^{-1}= \pm 0.076924423$. Using this result one can exactly generate also the coefficients 494 respectively 1368 as

$$
\begin{gathered}
\frac{2}{9} \cdot 13 \cdot\left(x+x_{1}^{-1}\right)^{2}=494 \\
8 \cdot\left(x+x_{1}^{-1}\right)^{2}=1368
\end{gathered}
$$

In contrast, when applying instead the quadratic equation $x^{2}+13 x-1=0$ similar to Equation (30), then this equation has solutions of $x_{1}=-\frac{1}{0.076473218}=-13.076473218$ and $x_{2}=0.076473218 \ldots$, which can also be tackled by an infinite continued fraction representation [58].

\section{About Double Well Potentials}

Because the polynomial $f(x)$ (Equation (1)) looks like an asymmetric double well potential curve, let us linger a little bit on such linear superposition of "classical" states as a concept important in quantum information theory [60].

On can suggest from the expression for the baryonic matter energy density [17] a quartic polynomial of a symmetric double-well form (Figure A1) with a maximum at $x=\varphi$

$$
p(x)=(x-1)\left(x-\varphi^{3}\right)\left(x-\varphi^{-1}+\frac{1}{13}\right)\left(x+\varphi^{2}-\frac{1}{13}\right)
$$

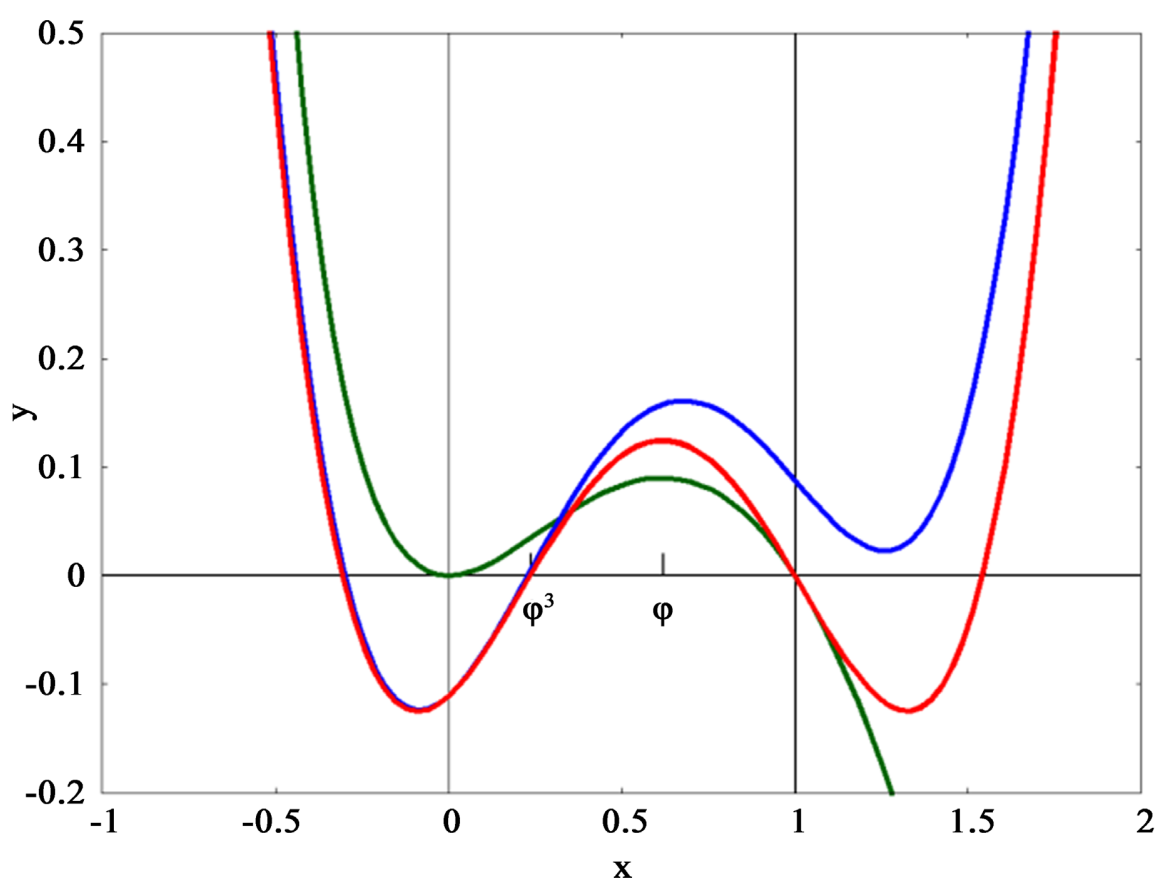

Figure A1. Illustration of the quartic polynomial $p(x)$ (red) in comparison with a slightly asymmetric variant (blue) and with Hardy's function (green). 


$$
p(x)=x^{4}-2.47213596 x^{3}+1.29382703 x^{2}+0.289285566 x-0.11097179
$$

where the coefficient of the $x^{3}$ term is $2\left(1+\varphi^{3}\right)$ and the last term can be approximated by $\frac{1}{13 \cdot \ln (2)}=0.1109765 \cdots$

Figure A1 depicts the quartic polynomial $p(x)$ besides an altered polynomial with a slightly increased coefficient of the quadratic term to get an asymmetrical double-well curve similar to $f(x)$. Both curves can be compared with the Hardy function [2] [17] indicating also a maximum at $x=\varphi$ and a high of $y_{\max }=\varphi^{5}$

$$
h(x)=(1-x) x^{2} /(1+x)
$$

One can alter the $p(x)$ polynomial to adapt well the physically relevant part of Hardy's function for $x \leq 1$. For instance, the following simple quartic double well polynomial maps the Hardy function well in the range $|x| \leq 0.2$ (see Figure A2) indicating its maximum at $x=1 / 2$ instead of $x=\varphi$ for the Hardy function

$$
q(x)=x^{4}-2 x^{3}+x^{2}
$$

When replacing the symmetric double well by an asymmetric curve according to

$$
q^{+}(x)=x^{4}-2 x^{3}+\left(1+\varphi^{5}\right) x^{2}
$$

then Hardy's functions can be well approximated still beyond the maximum exactly located at $x=\varphi$ with a high of $\varphi^{5}$.

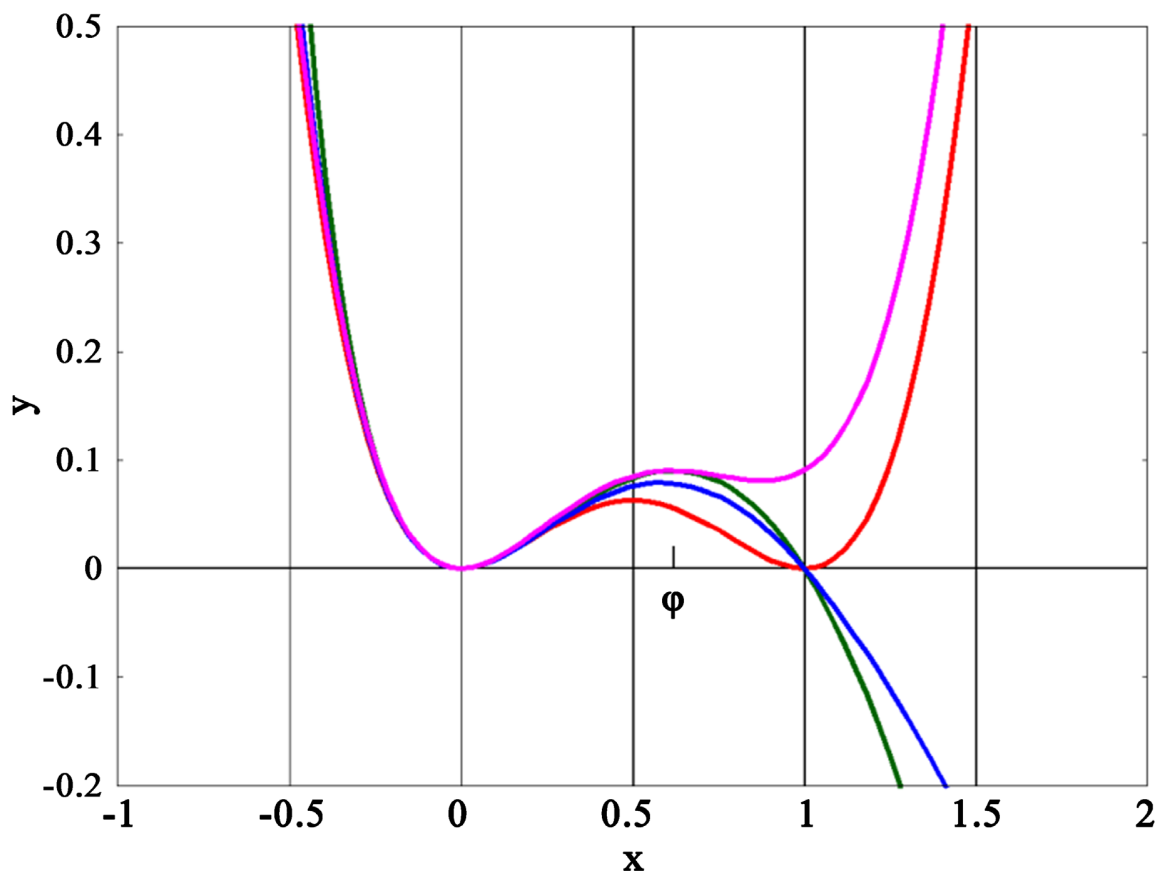

Figure A2. Double well polynomial $q(x)$ (red) in comparison with Hardy's function $h(x)$ (green) and with a first approximation according to Equation (53) in blue besides a second one according to Equation (52) in magenta. 
Another approximation was depicted in Figure A2 in blue concerning the Hardy function

$$
\tilde{h}(x)=(1-x) x^{2} \exp (-x)
$$

Instead of replacing $(1+x)^{-1}$ by $\exp (-x)$ [61] we have applied its series expansion, leading to the following excellent polynomial approximation

$$
\begin{aligned}
\tilde{h}(x) & \approx \sum_{n=1}^{\infty} x^{2} \cdot\left(\frac{1-x}{2}\right)^{n} \\
& =x^{2}\left(\frac{(1-x)}{2}+\frac{(1-x)^{2}}{4}+\frac{(1-x)^{3}}{8}+\frac{(1-x)^{4}}{16}+\frac{(1-x)^{5}}{32} \pm \cdots\right)
\end{aligned}
$$

which is depicted in Figure A3 in comparison with Hardy's function.

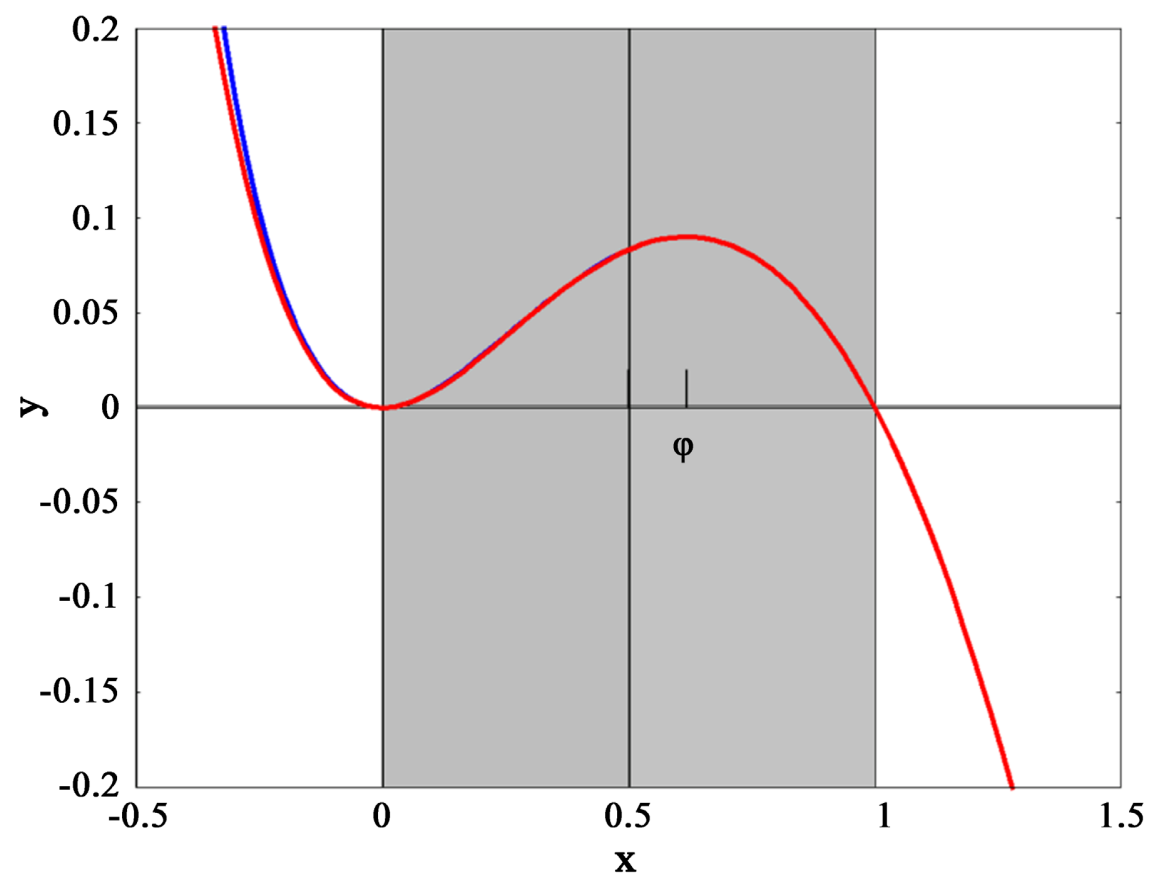

Figure A3. Polynomial approximation (red) of Hardy's function (blue) according to Equation (54). 